\title{
A First Look at Platinum Prints using Variable Pressure/Environmental High Resolution Scanning Electron Microscopy VP HR SEM
}

\author{
Patrick Ravines1, Natasha Erdman2, and Rob McElroy3 \\ 1Art Conservation Department, State University of New York College at Buffalo, \\ Buffalo, New York, USA \\ 2JEOL USA Inc., Peabody, MA, USA 01960 \\ 3Photographer \& daguerreotypist, Archive Studio, 347 Franklin St., Buffalo, NY 14202 \\ USA
}

This presentation will provide a first look at the nature of the image particles found on modern and historic platinum prints with the new line of variable pressure high-resolution scanning electron microscopes (VP HR SEM).

Modern advances in electron microscopy have made available instruments with variable and environmental pressures allowing for the study of organic materials such as cellulosic and proteinaceous materials without the need for sample preparation using carbon, gold or other metal coatings. This is advantageous since it then allows for direct observation of surfaces without interference from sample preparation protocols. Cross sections of modern and prepared using an Argon ion plasma polishing system have also been examined with the VP SEM.

The high resolution electron microscopic images of the modern print surfaces show evenly distributed and clumped, like grape bunches, nano-sized image particles ranging 'resting' on cellulose fibers. The pure platinum samples show even distribution of nanoparticles and the palladium-platinum samples show clumping. The image particle sizes in both modern samples are below $100 \mathrm{~nm}$ in size. Interestingly, the images of cross sections of the modern prints show that the nano-particles are embedded into the paper and cellulose fibers. The image particles are a few micrometers deep below the surface and appear to be distributed throughout the bulk of the paper. Within the fibers there seems to be a preferential formation/development of image particles between cell wall layers. Historic platinum prints are currently being studied with VP HR SEM for comparison with modern prints. 
Microsc. Microanal. 19 (Suppl 2), 2013 